\section{$\underset{\substack{\text { hommes } \\ \text { \& migrations }}}{ }$}

\section{Hommes \& migrations}

Revue française de référence sur les dynamiques

migratoires

$1302 \mid 2013$

Le Japon, pays d'immigration?

\title{
Histoire d'une immigration portugaise. Le rôle de la région Aquitaine
}

\author{
Manuel Dias Vaz
}

\section{(2) OpenEdition \\ Journals}

\section{Édition électronique}

URL : http://journals.openedition.org/hommesmigrations/2491

DOI : 10.4000/hommesmigrations.2491

ISSN : 2262-3353

\section{Éditeur}

Musée national de l'histoire de l'immigration

\section{Édition imprimée}

Date de publication : 1 avril 2013

Pagination : 154-156

ISBN : 978-2-919040-22-3

ISSN : $1142-852 X$

\section{Référence électronique}

Manuel Dias Vaz, « Histoire d'une immigration portugaise. Le rôle de la région Aquitaine », Hommes \& migrations [En ligne], 1302 | 2013, mis en ligne le 12 septembre 2013, consulté le 22 septembre 2020. URL : http://journals.openedition.org/hommesmigrations/2491 ; DOI : https://doi.org/10.4000/ hommesmigrations.2491 


\title{
L'ÉMIGRATION AU PORTUGAL \\ AVATAR D'UN PAYS "SEMI-PÉRIPHÉRIQUE", MÉTROPOLE POSTCOLONIALE
}

\author{
IRÈNE DOS SANTOS, docteure en anthropologie sociale de l'École des hautes \\ études en sciences sociales (Paris), chercheure postdoctorante au Centro em Rede \\ de Investigação em Antropologia (CRIA) de l'Université nouvelle de Lisbonne \\ et boursière de la Fondation pour la science et la technologie (FCT)
}

"C rise économique : 408 Portugais émigrent par jour r", "Guide pour émigrer2", "Portugais à la conquête du monde 3". L'émigration fait les gros titres des médias portugais et pourtant les spécialistes sont formels : contrairement à une idée répandue, une décélération de l'émigration a été observée depuis le début de la crise économique de 2008 et ce jusqu'à la fin de l'année $2011^{4}$.

Selon le sociologue Rui Pena Pires, coordinateur de l'Observatoire de l'émigration, les flux de sortie ont augmenté jusqu'en 2007 - 59912 départs annuels - et diminué à partir de 2008 - 53710 départs en 2008, 43225 en 20115. Cette décélération serait l'illustration de la nature globale de la crise qui atteint les pays de destination traditionnelle de l'émigration portugaise : France, Espagne, Royaume-Uni, Suisse, Allemagne, Luxembourg, et ces chiffres relativisent donc la situation.

Mais comment les choses ont-elles évolué depuis 2011, alors que l'accélération de la récession éco- nomique et l'austérité budgétaire imposée par la


Portugal, à un point tel que le gouvernement de Pedro Passos Coelho encourage depuis octobre 2011 les jeunes chômeurs - 38,2 \% des 18/25 ans 7 à émigrer ?

Selon les médias, depuis 2008, l'émigration s'est accélérée vers la France, le Brésil, l'Angola, le Royaume-Uni, la Suisse, le Canada, l'Allemagne, les États-Unis, la Belgique, le Luxembourg, le Venezuela, I'Inde... 600 ooo Portugais auraient ainsi émigré entre 2009 et 2012, soit une moyenne annuelle de 150 ooo départs, selon l'hebdomadaire Visão ${ }^{8}$ : le double des années 2000 et une moyenne annuelle bien supérieure au taux d'émigration des années $1965-1975^{9}$, sous la dictature salazariste. Mais, alors que l'Observatoire de l'émigration avance le chiffre de 708 entrées de Portugais au Brésil pour 2011, 2247 pour 2012, le journal Correio da Manhã $\tilde{a}^{10}$ publie lui le chiffre

1. João Saramago, "Por dia emigram 408 portugueses", in Correio da manhã, 6 décembre 2011. 2. Paulo M. Santos, "Guia para emigrar", in Visão, 15 novembre 2012, pp. 52-62. 3. "Portugueses à conquista do mundo : as vidas, as histórias e as ideias de quem está além-fronteiras", in Visão, numéro spécial 20 ans, 21 mars 2013. 4. Rui Pena Pires, "Emigração parece que aumenta porque chegou às 'famílias que contam”", in Diário de Notícias, 18 novembre 2012 [en ligne : http://www.dn.pt/inicio/portugal/interior.aspx?content_id=2893854; Jorge de Portugal Branco, "Novas chegadas de Portugueses a França travaram tendência de diminuição da Comunidade”, in Luso Jornal, dossier : " Onde estão os Portugueses de França ?", 9 janvier 2013, pp. 12-14. 5. http://www.observatorioemigracao.secomunidades.pt 6. Nom donné au trio Fonds monétaire international, Banque centrale et Commission européennes. La société portugaise vit au rythme des "visites" très médiatisées de surveillance de ces institutions internationales. 7. Eurostat, 2013 [en ligne : http://www.touteleurope.eu/fr/actions/social/emploi-protection-sociale/ presentation/comparatif-le-taux-de-chomage-des-jeunes-dans-l-ue.html, consulté le 15/04/2013]. 8. Visão, numéro spécial, op. cit. 9. 122000 départs annuels. Voir Maria loannis Baganha, “La emigración portuguesa”, in António Costa Pinto (dir.), Portugal contemporáneo, Madrid, Sequitur, 2000, pp. 187-204. 10. João Saramago, “Por dia emigram 408 portugueses”, art. cit. 
REPÉRAGE

de 52000 entrées pour les six premiers mois de l'année, en citant les données du ministère brésilien de la Justice.

\section{Enjeux politiques autour des chiffres}

La quête de données statistiques fiables et comparables tourne vite au casse-tête, un problème récurrent dans les études sur l'émigration compte tenu de la diversité des méthodes de calcul selon les pays d'installation", de la libre circulation au sein de l'espace de Schengen (l'émigration 'réelle' ne pouvant qu'être estimée),

Selon les médias, depuis

2008, l'émigration s'est accélérée vers la France, le Brésil, l'Angola, le Royaume-

Uni, la Suisse, le Canada, l'Allemagne, les États-Unis, la Belgique, le Luxembourg,

le Venezuela, l'Inde...

600000 Portugais auraient ainsi émigré entre 2009 et 2012 et aussi de l'instrumentalisation politique de l'émigration. L'Observatoire de l'émigration a été créé en 2008 par le secrétariat d'État aux Communautés portugais (ministère des Affaires étrangères) pour montrer la stabilité des flux migratoires, dans un contexte où l'opposition de centre droit (PSD, aujourd'hui au pouvoir) faisait de l'accélération de l'émigration une preuve de l'échec de la politique menée par le gouvernement de José Socrates ${ }^{12}$.Or, lorsqu'il s'agit d'évoquer la "diaspora", ou "les communautés portugaises", devenues une réalité distincte de "l'émigration", ce sont des chiffres - précis, mais là encore des estimations - en progression constante : 4 millions d'individus dans les années 1990, 5,7 millions à la fin des années 2000, qui sont convoqués pour signifier l'importance de cette nation "hors frontière13". L'envoi de devises au pays d'origine et le rôle de lobbying économique et culturel constituent des fonctions 'naturelles' qui sont sans cesse rappelées aux Portugais de l'étranger et à leurs descendants (les "Luso-descendants")'14.

Autre enjeu autour des chiffres, l'entrée du Portugal dans la forteresse européenne a placé le pays dans une position contradictoire ${ }^{15}$. Son adhésion à l'espace Schengen a entraîné la mise en place d'une politique restrictive d'immigration,ycompris à l'égard des originaires des anciennes colonies ${ }^{16}$. Or, dans le même temps, le Portugal redéfinissait ses relations avec ces pays, avec la création en 1996 d'une institution linguistique supranationale, la Communauté des pays de langue portugaise (CPLP17), qui prévoyait des liens de réciprocité dans les définitions des statuts de national et de citoyen.

\section{Émigration structurelle ou conséquence de la crise socio-économique?}

Depuis la fin du XIXe siècle, l'émigration a permis de réguler le système socio-économique portugais. Elle a été une "soupape de sûreté" pour atténuer

\footnotetext{
11. Jorge de Portugal Branco, “Portugueses em França (1980-2000). Uma comunidade integrada?”, in Maria Beatriz Rocha-Trindade (dir.), Migrações - Permanências e Diversidades, Lisbonne, Cemri/Afrontamento, 2009, pp. 85-129. 12. Gouvernement qui démissionnera en 2011 après avoir finalement demandé l'intervention du Fonds monétaire international, ce qui a conduit à des élections législatives anticipées. 13. Irène Dos Santos, Eduardo Caetano da Silva, “A mesma juventude noutra latitude': Iusodescendentes do Brasil e da França frente ao projeto nacional das 'comunidades portuguesas"', in Daniel Melo, Eduardo Caetano da Silva (dir.), Construção da nação e associativismo na emigração portuguesa, Lisbonne, Impresa de Ciências Sociais, 2009, pp. 125-178. 14. Ibid. ; Irène Dos Santos, "Les Luso-descendants : une nouvelle première génération d'émigrants ?”, in Agora débats/jeunesse, n²4, dossier : “Jeunes générations en Europe : regards croisés Est-Ouest”, 2007, pp. 44-54. 15. Bela Feldman-Bianco, "Brazilians in Portugal, Portuguese in Brazil: Constructions of Sameness and Difference", in Identities. Global Studies in Culture and Power, vol. 8, $\mathrm{n}^{\circ}$ 4, 2001, pp. 607-650. 16. En 2007, les étrangers originaires de ce pays composaient la moitié de la population étrangère au Portugal : $24 \%$ de Brésiliens (soit 107000 individus), suivis des Cap-Verdiens (12\%), des Angolais (6\%) et des Guinéens (6\%) : Serviço de Estrangeiros e Fronteiras, Relatório de Imigração Fronteiras e Asilo, 2008 (polycopié).

17. Angola, Brésil, Cap-Vert, Guinée-Bissau, Mozambique, São Tomé-et-Principes, Timor-Est.
} 
les tensions sociales à la fin du XIX $\mathrm{X}^{\mathrm{e}}$ siècle, avant d'être quasi interdite sous la dictature tout en étant une importante source de devises pour l'économie du pays ${ }^{18}$.

Bien que le Portugal soit devenu un pays d'immigration à partir des années 1980, l'émigration est restée une "constante structurelle19", quoique passée sous silence, car vécue comme le stigmate d'un pays certes européen mais "semi-périphérique ${ }^{20}$ ". La Une de l'hebdomadaire Visão - "Portugais à la conquête du monde ${ }^{21 "}$-, paradigmatique d'une "réinvention constante du rôle légendaire accompli par les navigateurs portugais à l'époque des grandes explorations maritimes ${ }^{22}$ " et d'une essentialisation de la capacité des Portugais à émigrer, illustre la difficulté à interroger les causes profondes d'une émigration structurelle séculaire. Rui Pena Pires attire d'ailleurs l'attention sur la distinction à faire entre le rôle joué par la crise, argument aujourd'hui avancé par le gouvernement pour inciter la jeunesse à émigrer, et le retard structurel découlant d'une absence séculaire d'investissements productifs, qui contraint non plus seulement les classes populaires mais aussi une main-d'œuvre qualifiée à émigrer ${ }^{23}$. La visibilité accordéeaujourd'huiàl'émigrationtiendraitmoins à une question de volume des flux qu'à la médiatisation d'une problématique jusqu'alors refoulée : "Pour la première fois, la nécessité d'émigrer touche des groupes sociaux ayant une plus grande qualité de vie, davantage accès à l'information, une plus grande influence, raison pour laquelle l'émigration est devenue un problème public ${ }^{24}$." Loin des chiffres, les témoignages rapportent chaque jour les nombreux départs, notamment vers l'Angola (23 787 entrées en 2009), le Brésil (2 247 en 2012) et le Mozambique25. Des pays qui frappent les imaginaires car ils sont associés à l'histoire coloniale du Portugal et de manière générale au renversement des rapports Nord-Sud : "L’Angola au secours du Portugal. La revanche de l'ancien colonisé", titrait Le Monde diplomatique ${ }^{26}$. Dans le cas portugais, ce renversement revêt deux
La visibilité accordée aujourd'hui à l'émigration tiendrait moins à une question de volume des flux qu'à la médiatisation d'une problématique jusqu'alors refoulée. aspects simultanés : des investissements économiques massifs de l'Angola au Portugal dans le secteur bancaire, les télécommunications et les médias ; l'émigration vers l'Angola de Portugais, non plus seulement des classes populaires, mais aussi des classes moyennes, "dont une partie a longtemps posé un regard condescendant sur les émigrés des années 1960-1970 27".

\section{Émigrer en Angola : "pour une vie meilleure" ?}

L'Angola, la plus ancienne et la plus grande colonie africaine de peuplement du Portugal, vers laquelle les émigrants-colons ont rechigné à partir dans les années 1950-1960, est aujourd'hui un pays en voie de développement qui connaît une croissance économique de plus de 7 \%, grâce notamment à la manne pétrolière (qui ne profite 


\section{REPÉRAGE}

pas à l'ensemble de la population, loin s'en faut). À l'indépendance, en 1975, de petits entrepreneurs portugais, commerçants et industriels, généralement des hommes mariés avec des femmes autochtones, sont restés dans le pays, et d'autres y sont venus pour investir avec le soutien de ces réseaux familiaux locaux. En
En Angola, les Portugais

- une population aux profils très hétérogènes constituent aujourd'hui

la troisième population étrangère, après les Chinois et les Brésiliens. 2002 , lors de la fin de la guerre civile, c'est sur ces liens transnationaux, sociaux et économiques directement issus de l'histoire coloniale que les flux migratoires du Portugal vers l'Angola ont partiellement pris appui : des ouvriers travaillant pour les entreprises portugaises de construction civile, une population masculine qui ne s'est pas installée durablement dans le pays, des cadres "expatriés" et des petits entrepreneurs transnationaux. Au bar d'un hôtel de Luanda, la capitale, j'assiste à l'automne 2012 à une discussion animée entre deux Portugais et un parent 'local' (un métis) qui tente de les convaincre de venir investir: "En neuf ans l'Angola s'est plus developpé que le Portugal en trente-cina $a_{n s^{28}}$ !" António est le chef cuisinier de l'un des restaurants portugais chics de Luanda qui vient d'être ouvert par un entrepreneur portugais présent depuis les années 1975. II a émigré en 2006 suite à l'échec de deux projets professionnels liés à la restauration, alors que la situation économique portugaise se détériorait rapidement. António est né en Angola en 1972, tout comme sa propre mère née en 1951 (de mère autochtone). La famille a fait partie des quelque 500000 "retornados", ces rapatriés d'Angola et du Mozambique, entre 1974 et $1975^{29}$. António a profité de ses relations familiales sur place pour trouver du travail.
Il espère que l'acquisition de la nationalité angolaise lui permettra une intégration (économique) plus rapide ${ }^{30}$. II n'interprète pas son émigration comme un retour dans le lieu d'origine, mais comme son unique chance de connaître une réussite socio-économique que la société portugaise n'est plus en mesure de lui proposer. Il envisage de faire venir sa femme et ses enfants dès qu'il aura les moyens de leur offrir "une vie agréable", ce qui à Luanda, une des villes les plus chères au monde, signifie un logement confortable dans une copropriété sécurisée et équipée - l'accès à l'eau et à l'électricité étant très problématique et inégalement réparti -, une école internationale pour ses enfants et une voiture haut de gamme. Pour le moment, il vit dans un logement attenant au restaurant, un petit bungalow moderne construit par son patron, "pour vivre en autarcie".

La majorité de ses collègues, des cadres de la restauration embauchés directement au Portugal, célibataires, n'envisagent pas de rester en Angola. La viey est considérée comme trop chère et surtout le pays serait "peu accueillant": "L'Angola a besoin de cadres qualifiés mais les Portugais n'y sont pas les bienvenus." Ces migrants ont l'intention de rentrer après avoir suffisamment épargné, à l'instar de l'émigration portugaise intra-européenne des années 1960-1970, avec comme but de devenir propriétaires et de rembourser les emprunts, le cas échéant.

Nuno, un architecte de 38 ans, a émigré en Angola en 2002, après avoir travaillé au Mozambique et à Dubaï. Marié en secondes noces à une Brésilienne rencontrée à Luanda, il vit dans une de ces nouvelles résidences luxueuses du sud de la capitale. Pour les cadres qualifiés, expatriés d'entreprises portugaises et multinationales, le coût de la vie très élevé est directement pris en charge par les

28. Les citations sont tirées d'une enquête ethnographique menée entre octobre et novembre 2012 à Luanda auprès d'une vingtaine d'individus. 29. Rui Pena Pires, et al., Os Retornados: um estudo sociográfico, Lisbonne, Instituto de Estudos para o Desenvolvimento, 1994. 30. Depuis le début des années 2010, face à l'augmentation des demandes émanant de Portugais, les exigences pour l'acquisition de la nationalité angolaise, qui permet la double nationalité, se sont durcies. 
entreprises. Ils vivent entre eux, mais aussi avec des Brésiliens et des Angolais qui ont le même pouvoir d'achat et qu'on rencontre les week-ends dans les restaurants et plages privées de l'île de Luanda. Ces migrants cherchent à se distinguer explicitement des "purs émigrants qui décident de partir en quête d'une vie nouvelle".

En Angola, les Portugais - une population aux profils très hétérogènes - constituent aujourd'hui la troisième population étrangère, après les Chinois et les Brésiliens. 21000 en 2003, ils seraient aujourd'hui près de 100 ooo, moitié moins selon les autorités angolaises qui ne comptabilisent ni les binationaux ni les illégaux. Le sujet est sensible. L'octroi de visas, censé être facilité depuis l'accord de l'automne 2011, continue de subir les aléas des relations diplomatiques tendues entre les deux pays, sur fond de complexes réciproques d'anciens colonisés/anciens colonisateurs en crise, et de scandales de corruption, Angolais et Portugais s'accusant par presse interposée de "racisme et d'hypocrisie" ("les élites portugaises détestent l'Angola et sont envieuses ${ }^{31 "}$ ") ou de "racisme anti-Blanc". La protection du marché du travail angolais relève aussi de la crainte d'“une exportation du chômage" portugais ${ }^{32}$. Les autorités attendent, par ailleurs, le retour des
Angolais émigrés dans les années 1990 et celui des jeunes élites parties étudier à l'étranger (Portugal, Espagne, Brésil). Lorsqu'ils rentrent, ces jeunes Angolais sont très critiques à l'égard de cette présence "amie" mais étrangère, "revenue profiter du pays", entretenant les inégalités passées. À la Sonangola, entreprise publique chargée de l'exploitation et de la production de pétrole et de gaz naturel, pour un même poste, les Portugais (les Européens en général) ont un salaire supérieur à celui des Angolais...

À Lisbonne, devant le consulat angolais situé sur les docks d'Alcântara, chaque jour la file d'attente semble un peu plus longue ${ }^{33}$. Mais le temps où l'Angola a pu constituer une destination privilégiée pour des jeunes diplômés et des petits entrepreneurs transnationaux ayant des liens familiaux est peut-être passé. Pour le moment, la majorité des nouveaux émigrés n'envisagent pas de s'installer dans la longue durée. Rentreront-ils rapidement ou finiront-ils, comme leurs prédécesseurs des XIX-XXe siècles, par s'y installer durablement en faisant venir leurs familles à l'instar d'António, ou en en fondant une sur place comme Nuno ? De nouveaux schémas migratoires peuvent aussi émerger dans un "espace lusophone" qui se reconfigure. 